\title{
Plasma phospholipid-mediated transfer of docosahexaenoic acid from liver to brain phospholipids in Type 1 and Type 2 diabetic rats
}

\author{
WILLY J. MALAISSE ${ }^{1}$, ABDULLAH SENER ${ }^{1}$ and YVON A. CARPENTIER ${ }^{2}$ \\ Laboratories of Experimental ${ }^{1}$ Hormonology and ${ }^{2}$ Surgery, Université Libre de Bruxelles, Brussels, Belgium
}

Received June 23, 2010; Accepted August 30, 2010

DOI: 10.3892/mmr.2010.360

\begin{abstract}
Docosahexaenoic acid (C22:6 13$)$ generated in the liver from circulating $\alpha$-linolenic acid (C18:3w3) was recently proposed as the source of brain $\mathrm{C} 22: 6 \omega 3$ when the latter fatty acid is absent from the diet. The present study aims at exploring whether a comparable situation prevails in Types 1 and 2 diabetic rats, even when exposed to a normal diet. The fatty acid profile of liver, plasma and brain phospholipids (PL) was examined in 22 rats including 6 streptozotocin-induced diabetic animals (Type 1 diabetes), 8 Goto-Kakizaki rats (Type 2 diabetes) and 8 control animals. Both fed and overnight starved rats of both genders were included in this study. To avoid the interference of any group effect, all individual results were expressed relative to the mean value found in animals of the same gender, same strain, same nutritional situation and same glycemic status, such results being referred to as normalized values. Significant positive correlations were observed between the normalized values for the $\mathrm{C} 22: 6 \omega 3$ relative weight content of liver and plasma PL and, after exclusion of 2 female control rats with an abnormally high brain/plasma PL C22:6w3 ratio, plasma and brain PL. Noticeably, the variation coefficients (SD/mean) for the 3 variables did not exceed $7.1 \pm 1.8 \%$. A comparable situation prevailed between liver, plasma and brain triglycerides. Our results suggest that circulating PL participate in the transfer of C22:6w3 from the liver to the brain, even in control and diabetic rats not deprived of a dietary source of long-chain polyunsaturated $\omega 3$ fatty acids.
\end{abstract}

\section{Introduction}

In a recent series of articles, Igarashi et al proposed that docosahexaenoic acid (C22:6w3) generated in the liver from

Correspondence to: Professor Willy J. Malaisse, Laboratory of Experimental Hormonology, Université Libre de Bruxelles, 808 Route de Lennik, B-1070 Brussels, Belgium

E-mail: malaisse@ulb.ac.be

Key words: Type 1 and Type 2 diabetic rats, docosahexaenoic acid, liver, plasma and brain phospholipids and triglycerides circulating $\alpha$-linolenic acid (C18:3 13$)$ is the source of brain $\mathrm{C} 22: 6 \omega 3$ when the latter fatty acid is absent from the diet (1-5). The major aim of the present study was to explore whether a comparable situation may prevail in control animals and both Type 1 diabetic rats (streptozotocin-induced diabetes; STZ rats) and Type 2 diabetic Goto-Kakizaki rats (GK rats). It involves 4 fed female control animals, 4 overnight starved female control animals, 6 fed female STZ rats, 4 fed male or female GK rats and 4 overnight starved GK rats also from both genders, in which the $\mathrm{C} 22: 6 \omega 3$ relative weight content of both phospholipids and triglycerides was measured in liver, plasma and brain.

\section{Materials and methods}

Full information on the metabolic status (6), fatty acid pattern of liver and plasma lipids $(7,8)$, as well as fatty acid pattern of brain lipids (9), of the 22 rats considered in the present study was previously provided in the cited articles.

Two types of comparison were conducted in the present study on the relative weight content of $\mathrm{C} 22: 6 \omega 3$ in liver, plasma and brain phospholipids or triglycerides. In the first instance, the absolute values for the $\mathrm{C} 22: 6 \omega 3$ relative weight content were taken into consideration. In the second instance, all results were expressed relative to the mean corresponding absolute value found in the same group of rats. This second approach is referred to as normalized values.

All results are expressed as mean \pm SEM (or individual difference from mean value whenever $n=2$ ). The statistical significance relative to the correlation coefficients was calculated by linear extrapolation between tabulated data.

\section{Results}

The C20:5 $\omega 3$ relative weight content of plasma phospholipids was below the limit of detection in 9 out of 22 rats, with an overall mean value of $0.197 \pm 0.040 \%(n=22)$. Likewise, the C20:5w3 relative weight content of brain phospholipids was below the limit of detection in 10 out of 22 rats, with an overall mean value not exceeding $0.021 \pm 0.004 \% \quad(n=22)$. No significant correlation was observed between these two variables $(\mathrm{r}=-0.0125 ; \mathrm{n}=22 ; \mathrm{p}>0.1)$. As illustrated in Fig. 1, a highly significant positive correlation was observed, however, 
Table I. Phospholipid and triglyceride C22:6w3 relative weight content in the liver, plasma and brain.

\begin{tabular}{|c|c|c|c|c|}
\hline & Liver & Plasma & Brain & $\mathrm{n}$ \\
\hline \multicolumn{5}{|c|}{ 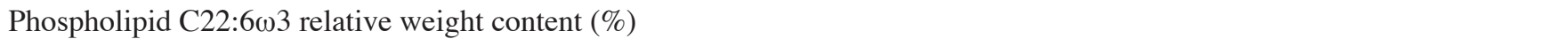 } \\
\hline Fed female control & $14.4 \pm 0.5$ & $6.9 \pm 0.2$ & $23.2 \pm 0.7$ & 4 \\
\hline Starved female control & $15.8 \pm 0.5$ & $8.4 \pm 0.2$ & $22.9 \pm 0.5$ & 4 \\
\hline Fed female STZ & $14.8 \pm 0.8$ & $6.9 \pm 0.2$ & $23.7 \pm 0.6$ & 6 \\
\hline Fed female GK & $18.8 \pm 1.8$ & $12.3 \pm 1.1$ & $27.0 \pm 1.8$ & 2 \\
\hline Starved female GK & $19.6 \pm 0.1$ & $13.1 \pm 0.2$ & $24.8 \pm 0.1$ & 2 \\
\hline Fed male GK & $12.5 \pm 1.5$ & $7.3 \pm 0.1$ & $24.7 \pm 0.2$ & 2 \\
\hline Starved male GK & $14.0 \pm 2.8$ & $9.2 \pm 0.7$ & $20.1 \pm 0.4$ & 2 \\
\hline \multicolumn{5}{|c|}{ Triglyceride $\mathrm{C} 22: 6 \omega 3$ relative weight content $(\%)$} \\
\hline Fed female control & $1.4 \pm 0.6$ & $2.3 \pm 0.3$ & $4.3 \pm 2.6$ & 4 \\
\hline Starved female control & $5.2 \pm 0.4$ & $9.5 \pm 1.3$ & $9.3 \pm 3.4$ & 4 \\
\hline Fed female STZ & $7.9 \pm 0.6$ & $7.4 \pm 0.4$ & $11.5 \pm 1.5$ & 6 \\
\hline Fed female GK & $4.6 \pm 0.1$ & $6.1 \pm 0.1$ & $6.5 \pm 4.9$ & 2 \\
\hline Starved female GK & $6.2 \pm 0.0$ & $10.8 \pm 0.1$ & $7.5 \pm 3.5$ & 2 \\
\hline Fed male GK & $6.8 \pm 1.1$ & $8.7 \pm 0.7$ & $2.6 \pm 0.7$ & 2 \\
\hline Starved male GK & $9.0 \pm 0.3$ & $11.6 \pm 0.4$ & $1.4 \pm 0.0$ & 2 \\
\hline
\end{tabular}

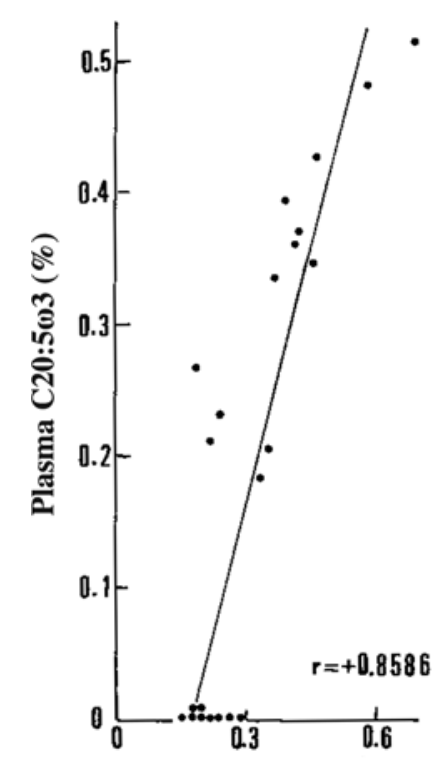

Liver C20:5 $\omega 3(\%)$

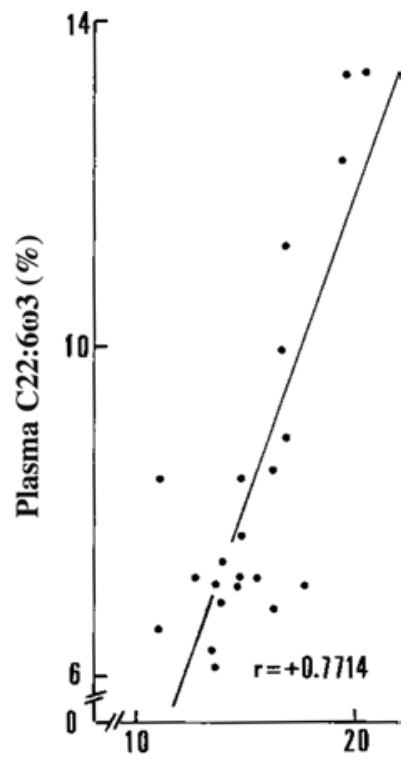

Liver C22:6 $\omega 3(\%)$
Figure 1. Correlation between the phospholipid relative weight content of $\mathrm{C} 20: 5 \omega 3$ (left; $\mathrm{n}=22$ ) and $\mathrm{C} 22: 6 \omega 3$ (right; $\mathrm{n}=22$ ) in the plasma and liver. The oblique lines correspond to the correlation lines with slope $\sqrt{\sum \mathrm{y}^{2} / \sum \mathrm{x}^{2}}$.

between the $\mathrm{C} 20: 5 \omega 3$ relative weight content of liver phospholipids, which yielded sizeable amounts of this fatty acid in all animals, and the C20:5 23 relative content of plasma phospholipids $(\mathrm{r}=+0.8586 ; \mathrm{n}=22 ; \mathrm{p}<0.001)$. Even when all individual results were expressed relative to the mean value recorded within the same group of rats, the correlation between such normalized values for the two variables under consideration remained highly significant $(r=+0.6622 ; n=22 ; p<0.001)$.

Likewise and as also illustrated in Fig. 1, a highly significant positive correlation prevailed between the $\mathrm{C} 22: 6 \omega 3$
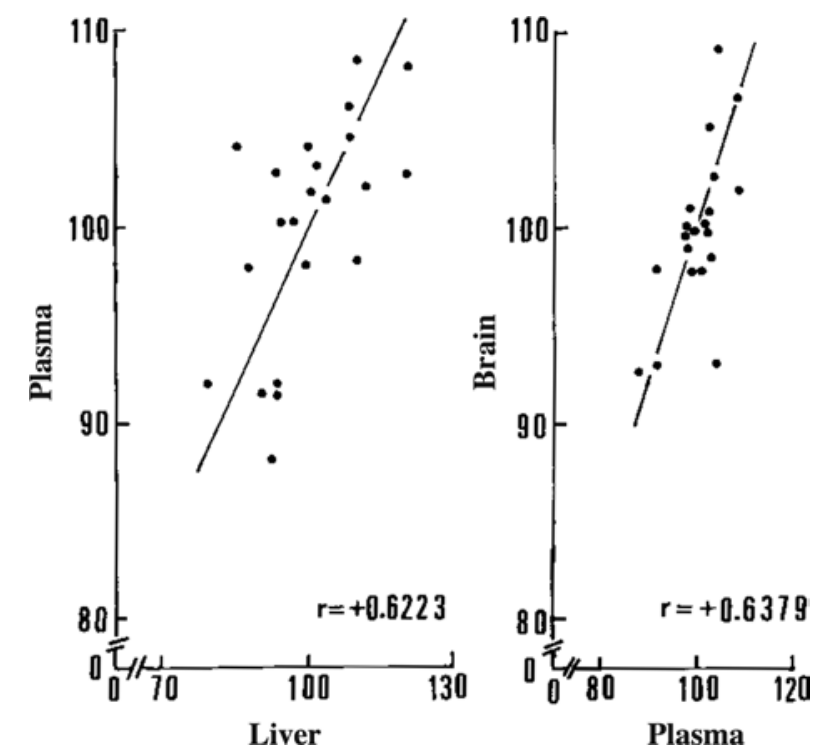

Figure 2. Correlation between the normalized values (\%) of plasma and liver (left; $n=22$ ) and brain and plasma (right; $n=20$ ) phospholipid C22:6 133 relative weight content. The oblique lines correspond to the correlation lines with slope $\sqrt{\sum \mathrm{y}^{2} / \sum \mathrm{x}^{2}}$.

relative weight content of liver and plasma phospholipids $(\mathrm{r}=+0.7714 ; \mathrm{n}=22 ; \mathrm{p}<0.001)$. Noticeably, the paired plasma/ liver ratios for the phospholipid relative content of $\mathrm{C} 22: 6 \mathrm{\omega} 3$ $(0.547 \pm 0.020 ; n=22)$ and $C 20: 5 \omega 3(0.517 \pm 0.101 ; n=22)$ were quite comparable $(\mathrm{p}>0.7)$ to one another.

The primary data for the $\mathrm{C} 22: 6 \omega 3$ relative weight content of liver, plasma and brain phospholipids are listed in Table I. The positive correlation between the absolute values for the liver and plasma phospholipid C22:6 03 content persisted when all individual measurements were expressed relative to the mean value found in the group of animals of same gender, 

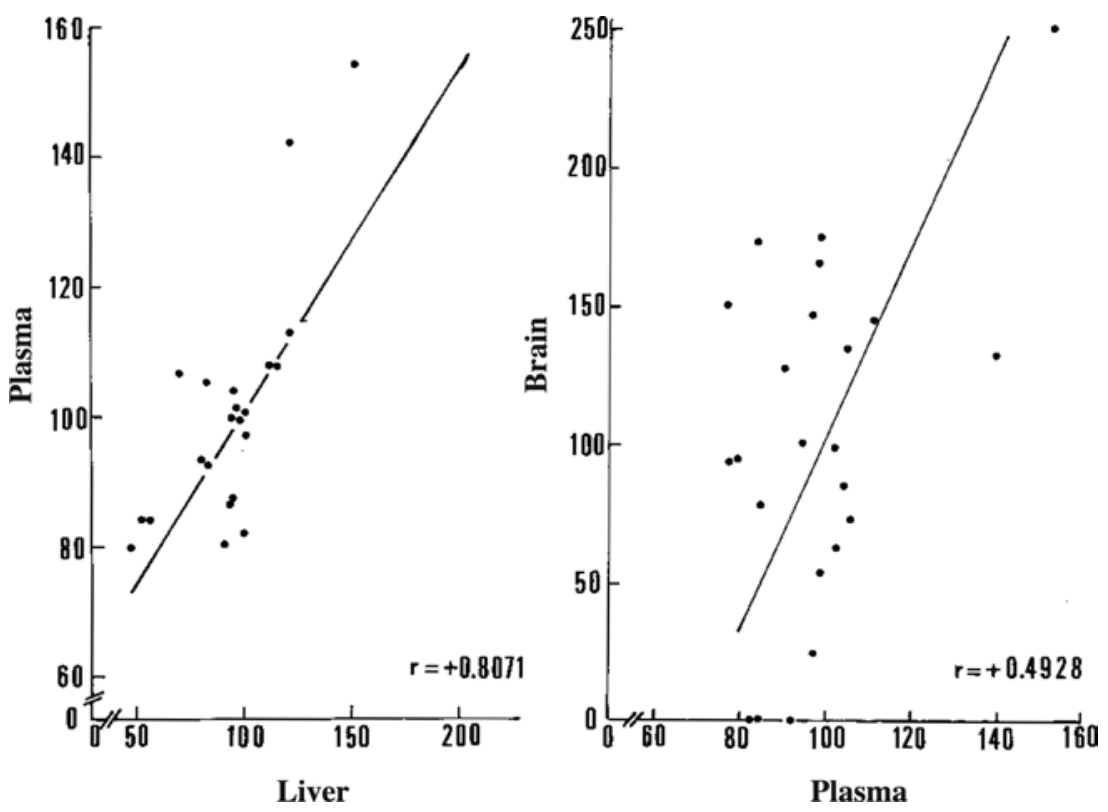

Figure 3. Correlation between the normalized values (\%) of plasma and liver (left; $n=22$ ) and brain and plasma (right; $n=22$ ) triglyceride C22:6 $\omega 3$ relative weight content. The oblique lines correspond to the correlation lines with slope $\sqrt{\sum \mathrm{y}^{2} / \sum \mathrm{x}^{2}}$.

same strain, same nutritional situation and same glycemic status $(r=+0.6223 ; n=22 ; p<0.004)$. As documented in Fig. 2, this indicates that such a correlation was not restricted to a group effect.

No significant correlation was found between the C22:6w3 relative weight content of plasma and brain phospholipids, whether the results were expressed in absolute terms $(\mathrm{r}=+0.3627 ; \mathrm{n}=22 ; \mathrm{p}>0.1)$ or as normalized values $(\mathrm{r}=+0.1794$; $\mathrm{n}=22 ; \mathrm{p}>0.1)$. One fed and one overnight fasted female control rat(s) displayed an abnormally high brain/plasma ratio for the $\mathrm{C} 22: 6 \omega 3$ relative weight content of phospholipids, representing $123.2 \pm 1.5 \%(n=2 ; p<0.001)$ of the mean corresponding values recorded in the other rats of the same group(s) $(100.0 \pm 1.1 \%$; $n=6$ ). In the analysis of the normalized values, these two female control rats also displayed an xy product well below the lower limit of the $95 \%$ confidence interval, as computed from the measurements made in the other 20 rats. After exclusion of these two female control rats, significant positive correlations were found between the $\mathrm{C} 22: 6 \omega 3$ relative weight content of plasma and brain phospholipids, whether the results were expressed in absolute terms $(\mathrm{r}=+0.5135 ; \mathrm{n}=20 ; \mathrm{p}<0.03)$ or as normalized values $(\mathrm{r}=+0.6379 ; \mathrm{n}=20 ; \mathrm{p}<0.005)$.

In considering the results illustrated in Fig. 2, it should be stressed that the three variables under consideration displayed rather low variation coefficients ( $\mathrm{SD} / \mathrm{mean}$ value) not exceeding $7.1 \pm 1.8 \%(n=3)$, with only 5 out of 66 measurements outside the restricted $90-110 \%$ range around the mean $100 \%$ value.

The possible contribution of plasma triglycerides in the transfer of $\mathrm{C} 22: 6 \omega 3$ from the liver to brain was also considered in the present study. Thus, the $\mathrm{C} 22: 6 \mathrm{\omega} 3$ relative weight content of liver, plasma and brain triglycerides was also measured (Table I). Highly significant positive correlations were found between the liver and plasma triglyceride $\mathrm{C} 22: 6 \omega 3$ relative weight content, whether considering the absolute values $(\mathrm{r}=+0.7249 ; \mathrm{n}=22 ; \mathrm{p}<0.01)$ or normalized values $(\mathrm{r}=+0.8071$; $\mathrm{n}=22 ; \mathrm{p}<0.001$ ) of these two variables (Fig. 3).

No significant correlation was observed between the absolute values of either liver or plasma triglyceride C22:6w3 relative weight content and brain triglyceride $\mathrm{C} 22: 6 \omega 3$ relative weight content, with respective correlation coefficients of $+0.3324(\mathrm{n}=22 ; \mathrm{p}>0.1)$ and $+0.1862(\mathrm{n}=22 ; \mathrm{p}>0.1)$. In contrast to these findings, significant positive correlations were found between the normalized values for liver and brain triglyceride C22:6 63 content $(\mathrm{r}=+0.6298 ; \mathrm{n}=22 ; \mathrm{p}<0.03)$, as well as plasma and brain triglyceride $\mathrm{C} 22: 6 \omega 3$ content $(\mathrm{r}=+0.4928 ; \mathrm{n}=22$; $\mathrm{p}<0.03)$.

No significant correlation was found between the C22:6w3 content of liver triglycerides and brain phospholipids, whether the results were expressed in absolute terms $(r=-0.1439 ; n=22$; $\mathrm{p}>0.1$ ) or as normalized values ( $\mathrm{r}=-0.0525 ; \mathrm{n}=22 ; \mathrm{p}>0.1$ ). Likewise, no significant correlation was observed between the C22:6w3 content of plasma triglycerides and brain phospholipids, whether the results were expressed in absolute terms $(\mathrm{r}=-0.1657 ; \mathrm{n}=22 ; \mathrm{p}>0.1)$ or as normalized values $(\mathrm{r}=+0.0555$; $\mathrm{n}=22 ; \mathrm{p}>0.1)$.

Sizeable amounts of long-chain polyunsaturated $\omega 3$ fatty acids in plasma unesterified fatty acids were only detected in the case of $\mathrm{C} 18: 3 \omega 3$ and $\mathrm{C} 22: 6 \omega 3$. Even so, no $\mathrm{C} 22: 6 \omega 3$ in plasma unesterified fatty acids could be detected in 7 animals (4 fed control female rats and 3 fed STZ female rats), which also failed to display any sizeable amount of $\mathrm{C} 18: 3 \omega 3$ in the same plasma unesterified fatty acids. The absolute or normalized values for the $\mathrm{C} 22: 6 \omega 3$ relative weight content in plasma unesterified fatty acids were compared to those in either plasma, liver or brain triglycerides and phospholipids and those in adipocyte lipids. Out of these 14 comparisons, only two yielded a significant correlation coefficient, namely those comparisons between the absolute values for the C22:6w3 relative weight content of plasma unesterified fatty 
acids and the $\mathrm{C} 22: 6 \omega 3$ relative weight content of either plasma triglycerides $(\mathrm{r}=+0.4920 ; \mathrm{n}=22 ; \mathrm{p}<0.04)$ or adipocyte lipids $(\mathrm{r}=+0.5151 ; \mathrm{n}=22 ; \mathrm{p}<0.02)$. The latter finding could reflect, to some extent at least, the modulation of lipolysis in adipocytes by such factors as starvation and diabetes. Indeed, and as an example, the ratio between the $\mathrm{C} 22: 6 \omega 3$ relative weight content of plasma unesterified fatty acids/adipocyte lipids was significantly higher $(\mathrm{p}<0.05)$ in overnight starved control female rats $(4.54 \pm 1.76 ; n=4)$ than in fed control female rats (zero value; $n=4$ ), with an in-between value in fed STZ female rats $(2.25 \pm 1.02 ; \mathrm{n}=6)$.

Some further quantitative aspects of the issue here under consideration should not be ignored. Expressed in absolute terms (mg/g tissue wet weight), the $\mathrm{C} 22: 6 \mathrm{\omega} 3$ content of lipids was highest $(\mathrm{p}<0.001)$ in adipose tissue $(11.54 \pm 1.02 ; \mathrm{n}=22$ in this and further cases), as compared to $3.64 \pm 0.15$ and $0.50 \pm 0.08$ in liver phospholipids and triglycerides, respectively, and $7.34 \pm 0.20$ and $0.04 \pm 0.00$ in brain phospholipids and triglycerides. In plasma, the values found in phospholipids $(106.9 \pm 11.1 \mu \mathrm{g} / \mathrm{ml})$ only represented approximately 2.9 and $1.5 \%$ of those found in liver and brain phospholipids, respectively. The $\mathrm{C} 22: 6 \omega 3$ content of plasma triglycerides $(\mu \mathrm{g} / \mathrm{ml})$ only represented $39.6 \pm 4.8 \%$ of the paired value found in plasma phospholipids, whilst that of plasma unesterified fatty acids $(3.80 \pm 0.63 \mu \mathrm{g} / \mathrm{ml})$ was further decreased to approximately $3.6 \%$ of that of plasma phospholipids. The latter finding again argues against any major role of circulating unesterified fatty acids to the supply of C22:6 63 to the brain, which yielded an absolute $\mathrm{C} 22: 6 \omega 3$ content in phospholipids about twice higher than in liver phospholipids (paired brain/ liver ratio: $2.099 \pm 0.121 ; \mathrm{n}=22 ; \mathrm{p}<0.001$ vs. unity).

\section{Discussion}

Docosahexaenoic acid (C22:6w3) is important for optimal brain function. Hence, the relative contribution of dietary

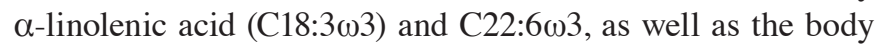
stores of phospholipids in long-chain polyunsaturated $\omega 3$ fatty acids, to the regulation of brain $\mathrm{C} 22: 6 \omega 3$ content represents a relevant issue (10). For instance, when C22:6w3 is absent from the diet, its hepatic generation from circulating $\mathrm{C} 18: 3 \omega 3$ was proposed to be the source of brain C22:6w3 (1-5).

The present study concerns mainly, but not exclusively, the possible role of circulating phospholipids in the supply of C22:6w3 from liver to brain in both normal and either Type 1 or Type 2 diabetic rats. In this perspective, the starting observation consisted in the significant correlation found in the 22 rats examined in this study between the individual values for the relative weight content of $\mathrm{C} 22: 6 \mathrm{\omega} 3$ in liver and brain phospholipids $(\mathrm{r}=+0.4299 ; \mathrm{n}=22 ; \mathrm{p}<0.05)$.

In this quite heterogeneous set of rats, in terms of gender, strain, nutritional status (fed or overnight fasted rats) and glucose homeostasis (euglycemic or diabetic rats), highly significant positive correlations were found between the absolute values for the $\mathrm{C} 20: 5 \omega 3$ or $\mathrm{C} 22: 6 \omega 3$ relative weight content of liver and plasma phospholipids. Moreover, the paired plasma/liver ratio for the relative weight content of C20:5 13 and $\mathrm{C} 22: 6 \mathrm{\omega} 3$ in phospholipids was closely comparable to one another. This suggests that circulating phospholipids display a $\omega 3$ fatty acid pattern similar to that of liver phospholipids.
Even when all individual measurements were expressed relative to the mean value found in the group of animals of same gender, same strain, same nutritional situation and same glycemic status, significant positive correlations persisted between such normalized values for the $\mathrm{C} 20: 5 \omega 3$ or $\mathrm{C} 22: 6 \omega 3$ relative weight content of liver and plasma phospholipids. This indicates that, under these stringent analytic conditions, the positive correlations documented an interrelation at the individual level, independently of any group effect.

After exclusion of two female control rats displaying an abnormally high brain/plasma ratio for the $\mathrm{C} 22: 6 \omega 3$ relative weight content of phospholipids, significant positive correlations were also observed between the $\mathrm{C} 22: 6 \omega 3$ relative weight content of plasma and brain phospholipids, whether the results were expressed in absolute terms or as normalized values. Such interrelations are even more striking, when considering that the dispersion of individual normalized values was quite restricted, with less than only $8 \%$ of such data outside the $90-110 \%$ range around the mean value.

Taken as a whole, these findings are compatible with the view that, even in animals not deprived of a dietary supply of long-chain polyunsaturated $\omega 3$ fatty acids, circulating phospholipids play a far-from-negligible role in the supply of C22:6 13 from liver to brain phospholipids.

A comparable situation prevailed in the case of liver, plasma and brain triglycerides. Thus, significant positive correlations were also found between the normalized values for the triglyceride relative weight content of $\mathrm{C} 22: 6 \omega 3$ in liver and plasma, liver and brain, and plasma and brain. No evidence was obtained, however, to suggest a participation of liver or plasma triglycerides to the supply of C22:603 to brain phospholipids.

It could be objected that two fed female control rats were excluded from the correlation between the $\mathrm{C} 22: 6 \omega 3$ relative weight content of plasma and brain phospholipids illustrated in Fig. 2. However, these 2 rats not only differed from the other animals in the same groups by an abnormally high brain/plasma ratio for the variables under consideration, but also by an abnormally high $\mathrm{C} 22: 6 \omega 3 / \mathrm{C} 20: 5 \omega 3$ ratio when comparing the measurements made in plasma and liver triglycerides. The triglyceride plasma/liver quotient for such a ratio was indeed $36.5 \pm 13.7 \%$ higher $(\mathrm{df}=5 ; \mathrm{p}<0.05)$ in these 2 female control rats $(136.5 \pm 13.3 \% ; n=2)$ than the mean reference values found in the other animals of the same groups $(100.0 \pm 7.1 \% ; n=5)$. Moreover, it was previously observed, in another homogeneous group of 16 fed female control rats, that 2 animals displayed an abnormally high plasma/liver ratio for the $\mathrm{C} 20: 5 \omega 3, \mathrm{C} 22: 5 \omega 3$ and $\mathrm{C} 22: 6 \omega 3$ relative weight content of triglycerides (11). Thus, an unusual pattern of long-chain polyunsaturated $\omega 3$ fatty acids in selected lipids in otherwise apparently normal rats does not seem to represent a quite exceptional situation.

It has been proposed that there is no evidence for direct incorporation of esterified palmitic acid from plasma into brain lipids of awake adult rat (12). In this prior study, concerned with the fate of palmitate and not docosahexaenoate, it was confessed, however, that, the experiments lasting only $2 \mathrm{~h}$, it remained possible that circulating lipids other than plasma unesterified fatty acids could contribute to a direct transfer of esterified palmitic acid to the brain. In the present study 
concerned with the fate of docosahexaenoic acid and conducted under close-to-steady state conditions, no indication was found to support a key role of plasma unesterified C22:6 63 in the supply of this long-chain polyunsaturated $\omega 3$ fatty acid to brain lipids. In this respect, a distinction ought probably to be made between plasma unesterified fatty acids and unesterified fatty acids generated from esterified fatty acids in the vicinity of relevant target cells.

In conclusion, the present data document, at the individual level and independently of any group effect, interrelations between the $\mathrm{C} 22: 6 \omega 3$ relative weight content of liver, plasma and brain phospholipids, as well as liver, plasma and brain triglycerides in both control and either Type 1 and Type 2 diabetic rats. Although the finding of a tight correlation between two variables is obviously not sufficient, when taken alone, to document a direct cause-to-effect link, our results suggest that circulating phospholipids and triglycerides contribute to the transfer of $\mathrm{C} 22: 5 \omega 3$ in the same class of lipids from the liver to brain.

\section{Acknowledgements}

We are grateful to C. Demesmaeker for the secretarial help.

\section{References}

1. Igarashi M, DeMar JC Jr, Ma K, Chang L, Bell JM and Rapoport SI: Upregulated liver conversion of alpha-linolenic acid to docosahexaenoic acid in rats on a 15 week n-3 PUFAdeficient diet. J Lipid Res 48: 152-164, 2007.

2. Igarashi M, DeMar JC Jr, Ma K, Chang L, Bell JM and Rapoport SI: Docosahexaenoic acid synthesis from alphalinolenic acid by rat brain is unaffected by dietary n-3 PUFA deprivation. J Lipid Res 48: 1150-1158, 2007.

3. Igarashi M, Ma K, Chang L, Bell JM and Rapoport SI: Dietary n-3 PUFA deprivation for 15 weeks upregulates elongase and desaturase expression in rat liver but not brain. J Lipid Res 48 2463-2470, 2007.
4. Rapoport SI, Rao JS and Igarashi M: Brain metabolism of nutritionally essential polyunsaturated fatty acids depends on both the diet and the liver. Prostaglandins Leukot Essent Fatty Acids 77: 251-261, 2007.

5. Gao F, Kiesewetter D, Chang L, Ma K, Bell JM, Rapoport SI and Igarashi M: Whole body synthesis-secretion rates of long-chain n-3 PUFAs from circulating unesterified alpha-linolenic acid in unanesthetized rats. J Lipid Res 50: 749-758, 2009.

6. Malaisse WJ, Portois L, Zhang Y, Oguzhan B, Louchami K, Jijakli H, Courtois P, Sener A and Carpentier YA: Fatty acid content and pattern of edipidymal and parametrial adipose tissue lipids in streptozotocin (type 1) and Goto-Kakizaki (type 2) diabetic rats. Int J Mol Med 18: 1231-1234, 2006.

7. Malaisse WJ, Portois L, Zhang Y, Oguzhan B, Louchami K, Jijakli H, Courtois P, Sener A and Carpentier YA: Anomalies of liver and plasma triglyceride and phospholipid fatty acid pattern in streptozotocin (Type 1) and Goto-Kakizaki (Type 2) diabetic rats. Res Rev Biosci 78-86, 2007.

8. Malaisse WJ, Portois L, Zhang Y, Oguzhan B, Louchami K, Jijakli H, Courtois P, Sener A and Carpentier YA: Perturbation of desaturase- and elongase-catalyzed equilibrium between fatty acids in liver and plasma triglycerides and phospholipids in streptozotocin (Type 1) and Goto-Kakizaki (Type 2) diabetic rats. Res Rev Biosci 87-98, 2007.

9. Malaisse WJ, Zhang Y, Louchami K, Sener A, Portois L and Carpentier YA: Brain phospholipid and triglyceride fatty acid content and pattern in Type 1 and Type 2 diabetic rats. Neurosci Lett 409: 75-79, 2006.

10. Lefkowitz M, Lim S-Y, Lin Y and Salem N Jr: Where does the developing brain obtain its docosahexaenoic acid? Relative contributions of dietary $\alpha$-linolenic acid, docosahexaenoic acid, and body stores in the developing rat. Pediatr Res 57: 157-165, 2005.

11. Carpentier YA, Portois L, Sener A and Malaisse WJ: Correlation between the liver and plasma acid profile of phospholipids and triglycerides. Int J Mol Med 22: 255-262, 2008

12. Purdon D, Arai T and Rapoport S: No evidence for direct incorporation of esterified palmitic acid from plasma into brain lipids of awake adult rat. J Lipid Res 38: 526-530, 1997. 